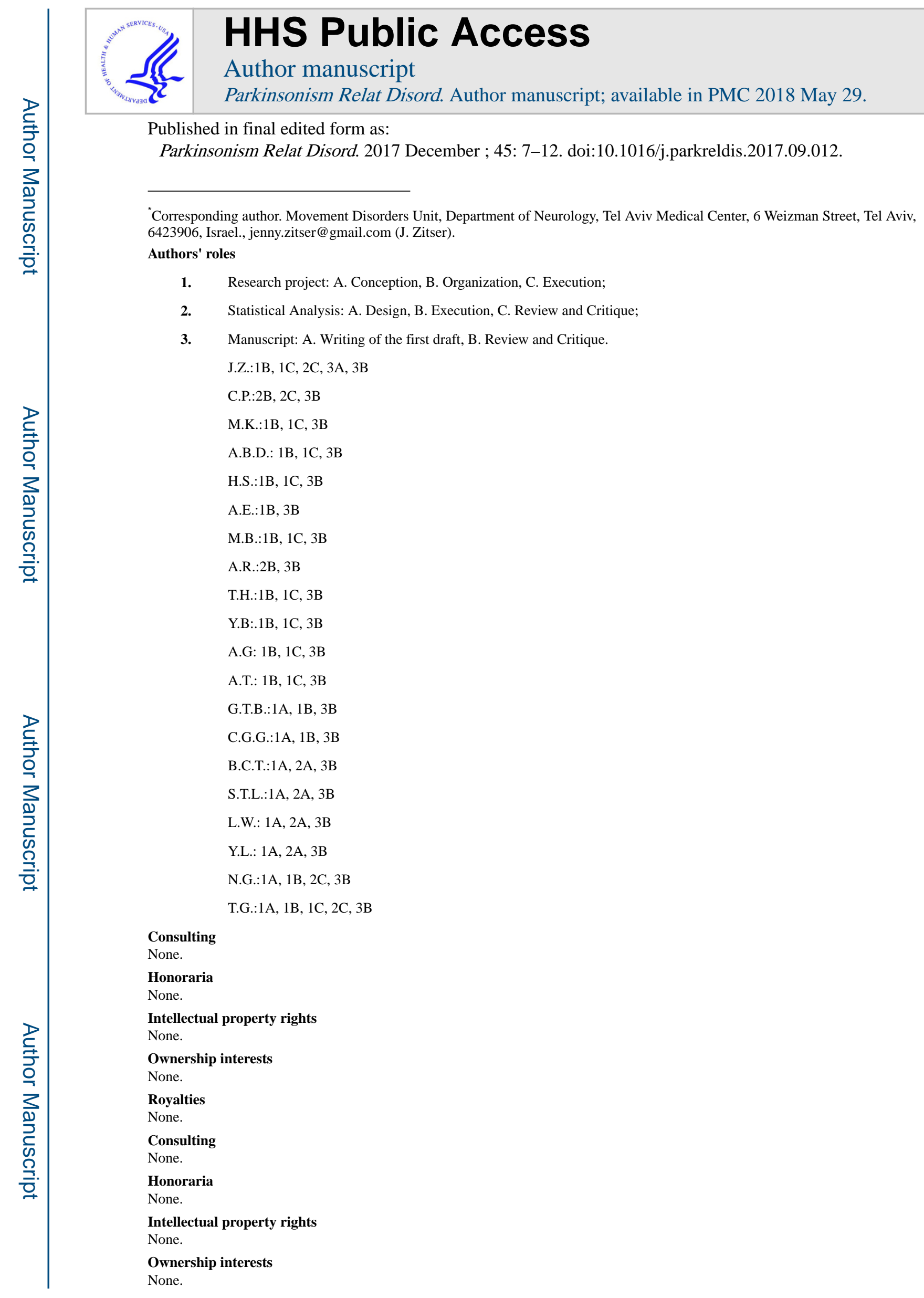




\title{
Validation of the Hebrew version of the Movement Disorder Society-Unified Parkinson's Disease Rating Scale
}

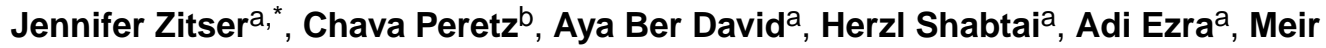 \\ Kestenbaum $^{\mathrm{a}}$, Marina Brozgol ${ }^{\mathrm{a}}$, Alina Rosenberg ${ }^{\mathrm{b}}$, Talia Herman ${ }^{\mathrm{a}}$, Yakov Balash ${ }^{\mathrm{a}}$, Avi \\ Gadoth $^{\mathrm{a}}$, Avner Thaler ${ }^{\mathrm{a}, \mathrm{e}}$, Glenn T. Stebbins ${ }^{c}$, Christopher G. Goetz ${ }^{\mathrm{c}}$, Barbara C. Tilley ${ }^{\mathrm{d}}$, \\ Sheng T. Luo ${ }^{d}$, Yuanyuan Liu ${ }^{d}$, Nir Giladia,e,f and Tanya Gurevich ${ }^{a, e, f}$ \\ aMovement Disorders Unit, Neurological Institute, Tel Aviv Medical Center, Tel-Aviv, Israel \\ bSchool of Public Health, Sackler School of Medicine, Tel-Aviv University, Tel-Aviv, Israel \\ 'Department of Neurological Services, Rush University Medical Center, Chicago, IL, USA \\ dDepartment of Biostatistics, School of Public Health, University of Texas Health Science Center \\ at Houston, Houston, TX, USA
}

Royalties

None.

Financial disclosures of all authors (for the preceding 12 months)

Nir Giladi - Serves as a member of the Editorial Board for the Journal of Parkinson's Disease. He serves as consultant to TevaLundbeck, IntecPharma, NeuroDerm, Armon Neuromedical LtdlDexel, Monfort, Lysosomal Therapeutics Inc. Accelmed Ltd, and Genzyme-Sanofi. He Received payment for lectures from Teva-Lundbeck, Novartis, UCB, Abbvie, Shaier and Genzyme. Prof. Giladi received research support from the Michael J Fox Foundation, the National Parkinson Foundation, the European Union 7th Framework Program and the Israel Science Foundation as well as from Teva NNE program, LTI, and Abbvie and CHDI.

Intellectual Property Rights: Tel-Aviv Medical Center,
parkinsonian monitoring by body fixed sensors of
motion and behavior, treatment and testing of gait and
falls using virtual reality,
Employment:
Contracts: None
Royalties: LTI

Other: Private donations to TLVMC, for research and patients care.
Stock Ownership in medically-related fields: in Lysosomal Therapeutics Ltd.

Advisory Boards: Teva-Lundbeck, LTI, Dexel, Abbvie, NeuroDerm, IntecPharma,
Genzyme-Sanofi, Accelmed Ltd
Partnerships: None
Honoraria: Teva-Lundbeck, Novartis, UCB, Movement Disorders Society,
Genzyme, Shire, Asian Neuro-rehabilitation Society

Grants: Michael J Fox Foundation, the National Parkinson Foundation, Parkinson Disease Foundation, CHDI the European Union 7th Framework Programme, the Israel Science Foundation, TEVA Innovative Ltd. NNE

Tanya Gurevich - Receives research support from the National Parkinson's Disease Foundation, and Jacques \& Gloria Gossweiler Foundation. She served at the advisory board of Abbvie Israel. She received honoraria from Abbvie and Novartis. She and her staff received support for the participation in scientific meetings from Teva, Medisson, Abbvie, Allergan, Medtronic, Megapharm, Novartis and Neuroderm companies.

Christopher G.-

Goetz - Barbara C. Tilley-

Sheng T. Luo-

Salary

University of Texas School of Public Health: (MDS support for project included)

Yuanyuan Liu-

Salary

University of Texas School of Public Health (MDS support for project included); UTHealth Innovation for Cancer Prevention Research Training Program Predoctoral Fellowship (Cancer Prevention and Research Institute of Texas grant \# RP160015)

J.Z, C. P, M. K, A.B.D, H.S., A.E., M.B., A. R., T. H., Y. B., A.G., A.T. have no financial disclosures. 
eSackler School of Medicine, Tel-Aviv University, Tel-Aviv, Israel

fSagol School of Neuroscience, Tel-Aviv University, Tel-Aviv, Israel

\section{Abstract}

Background-The Movement Disorders Society (MDS) published the English new Unified Parkinson's Disease Rating Scale (MDS-UPDRS) as the official benchmark scale for Parkinson's disease (PD) in 2008. We aimed to validate the Hebrew version of the MDS-UPDRS, explore its dimensionality and compare it to the original English one.

Methods-The MDS-UPDRS questionnaire was translated to Hebrew and was tested on 389 patients with PD, treated at the Movement Disorders Unit at Tel-Aviv Medical Center. The MDSUPDRS is made up of four sections. The higher the score, the worst the clinical situation of the patient is. Confirmatory and explanatory factor analysis were applied to determine if the factor structure of the English version could be confirmed in the Hebrew version.

Results-The Hebrew version of the MDS-UPDRS showed satisfactory clinimetric properties. The internal consistency of the Hebrew-version was satisfactory, with Cronbach's alpha values $0.79,0.90,0.93,0.80$, for parts 1 to 4 respectively. In the confirmatory factor analysis, all four parts had high (greater than 0.90) comparative fit index (CFI) in comparison to the original English MDS-UPDRS with high factor structure (0.96, 0.99, 0.94, 1.00, respectively), thus confirming the pre-specified English factor structure. Explanatory factor analysis yielded that the Hebrew responses differed from the English one within an acceptable range: in isolated item differences in factor structure and in the findings of few items having cross loading on multiple factors.

Conclusions-The Hebrew version of the MDS-UPDRS meets the requirements to be designated as the Official Hebrew Version of the MDS-UPDRS.

\section{Keywords}

Parkinson's disease; Rating scales; Clinimetrics; MDS-UPDRS; Validation

\section{Introduction}

Parkinson's disease (PD) is a progressive disease that affects movement and many non-motor systems [1]. There is currently no cure for PD. It is believed that the natural course of the disease can be modified by treatment, however, therapeutic interventions can also be associated with both motor and non-motor complications. Since there is no biomarker or other gold standard index to determine the severity of PD as a whole, the severity of each of the different PD elements is assessed separately, based on clinical rating scales. The most common scale in current use is the Unified Parkinson's Disease Rating Scale (UPDRS) [2], which is applied to follow the longitudinal disease course. After its introduction in the 1980s, the UPDRS became the gold standard clinical rating scale for PD [3,4]. In 2008, a new Movement Disorders Society (MDS)-sponsored revision of the UPDRS, known as the MDS-UPDRS, successfully passed clinimetric testing with high internal consistency and reliability for each of its parts [5]. Specifically, the MDS-UPDRS is made up of four sections [6]: Part I: evaluation of mentation, behavior, and mood; Part II: self-evaluation of 
the activities of daily life (ADLs), including speech, swallowing, handwriting, dressing, hygiene, falling, salivating, turning in bed, walking, cutting food; Part III:clinician motor examination of parkinsonism signs including the Hoehn \& Yahr staging of severity of Parkinson's disease [7]; Part IV: evaluation of motor fluctuations, dyskinesia and dystonia. The MDS-UPDRS use scores rated by both the patient and the physician to assess the gravity of the disease and the accompanying symptoms. It helps the clinician to stage the disease. The six items that deal with complex behaviors in Part I and all of the items that relate to fluctuations and dyskinesia in Part $I V$ require rater (clinician) led interviews of the patient, caregiver or both. The remaining seven questions of Part I as well as all items in Part $I I$ are answered with a straight-forward patient questionnaire and do not require any input from the rater [5].

The MDS-UPDRS was specifically designed to be less ambiguous than its predecessor, and the major patient/caregiver involvement means that it is vital that they understand the questions, preferably in their mother tongue. Evidence about the achievement of that objective requires future studies directly comparing both scales. The MDS-UPDRS has 50 items ( 65 scores) while the UPDRS has 42 items ( 55 scores). The apparent disadvantage of the MDS-UPDRS being longer to administer becomes inconsequential given the amount and quality of the data it provides. The average time for its administration is around $30 \mathrm{~min}$ [5]. In order to successfully translate and be designated as an "Official MDS translation" in a foreign language, the MDS has set strict protocol and criteria for cross validating nonEnglish versions of the MDS-UPDRS. Traditional Chinese, Estonian, French, German, Greek, Hungarian, Italian, Japanese and Korean versions are currently available [8]. We aimed to validate the Hebrew version of the MDS-UPDRS, to explore the dimensionality of the Hebrew version, and to compare it to that of the English version.

\section{Materials and methods}

\subsection{Translation of the MDS-UPDRS}

The process of developing an officially approved translation of the MDS-UPDRS involves five core steps: (1) registration and startup; (2) translation and independent back-translation; (3) cognitive pretesting to establish that the translation is clear and that it is comfortably administered to and completed by native-speaker raters and patients; (4) field testing in the native language using a large sample of Parkinson's disease patients; and (5) full clini-metric testing [9].

The cross cultural validation was performed as follows: First, the MDS-UPDRS was translated into Hebrew by an expert translation company; Afterwards it was reviewed and modified for an appropriate level of language by a team of PD experts in Israel led by Nir Giladi; It was then back-translated to English by the expert translation company (who were fluent in English and Hebrew and not involved in the original translation). This backtranslation was reviewed by a team of US experts (Glenn Stebbins, Christopher Goetz, Nancy LaPelle, and Barbara Tilley) who had been involved in the development of the original American version [6]. 


\subsection{Cognitive pretesting}

We constructed a qualitative structured interview format to evaluate specific items of the MDS-UPDRS in terms of task difficulty for the examiner and the respondent and the respondent's interest, attention span, discomfort and comprehension. Items were selected for cognitive pretesting when the observed differences were between the back translated Hebrew version and the English version. In addition, specific items that were identified in cognitive testing of the American version were also tested [6]. Items had an ordinal scale of six responses; e.g. "how helpful are the instructions for the patient?" with responses ranging from $1=$ not helpful to $6=$ very helpful, or, "how difficult was it for you as the rater to select the appropriate response option?" very difficult $=1$ up to $6=$ very easy.

The items included in the cognitive pretesting were: Cognitive impairment, depression, anxious mood, handwriting, freezing, finger tapping, hand movements, leg agility, arising from chair, postural tremor, time spent with dyskinesias and functional impact of dyskinesias. A total of 25 patients with Parkinson's disease and their examiners were interviewed in three rounds using the type of structured interview format typical for cognitive pretesting between November 2011 to July 2013. The final version of the translation was determined when the cognitive pretesting had been completed free of any problems.

\subsection{Study population and examiners}

The participants were 389 patients from the Movement Disorders Unit of the Tel Aviv Sourasky Medical Center who were recruited between November 2014 and August 2015. Seven experienced Israeli movement disorder specialists were recruited to examine native Hebrew-speaking PD patients of all ages and at all disease stages.

\subsection{Statistical analysis}

The descriptive statistics included frequencies and summary measures, and the analytical statistics included factor analysis, both confirmatory factor analysis (CFA) and secondary exploratory factor analyses (EFA). . A CFA of the overall factor structure (assuming categorical scale of the questionnaire components) was conducted to determine if the factor structure for the English language MDS-UPDRS [5] could be confirmed in the Israeli data. The CFA was conducted separately for MDS-UPDRS Parts I to IV. We evaluated the CFA results based on the Comparative Fit Index (CFI), and a CFI for each Part (I-IV) of 0.90 or greater was considered as a successful translation compared to the English language version (Table 1). An EFA (with an orthogonal CF-VARIMAX rotation) was conducted on the MDS-UPDRS Parts I-IV to explore the underlying factor structure without the constraint of a pre-specified factor structure. We used a scree plot to choose the number of factors to retain for each MDS-UPDRS part [10]. Once the factors were chosen, an item was retained if the factor loading for that item was 0.40 or greater. Analysis was performed using M-plus, Version 7.4.

\subsection{Ethics}

The program for validation of the MDS-UPDRS Hebrew version and for the collection of human data were approved by the Ethics Committee of the Tel Aviv Sourasky Medical 
Center. All the patients gave their signed consent to participate after receiving the pertinent information. The data did not include the patients' names or medical record numbers, and they were transferred to the team for analysis via a secure web-site.

\section{Results}

\subsection{Study population characteristics}

The demographic characteristics of the sample are shown in Table 2A. The Israeli sample included 389 patients(mean age $69.1 \pm 9.4$ years, $58.6 \%$ males). They were all native Hebrew-speaking PD patients and, according to the Hoehn \& Yahr classification, 75 (19.3\%) were in stage 1, $134(34.5 \%)$ in stage 2, $121(31.1 \%)$ in stage 3, $49(12.6 \%)$ in stage 4 and 9 $(2.3 \%)$ in stage 5 . The distributions of the scores of the four parts of the questionnaire are presented in Table 2B.

\subsection{Confirmatory factor analysis (CFA)}

The CFA for all four parts of the Hebrew MDS-UPDRS was high in comparison to the English MDS-UPDRS factor structure (0.96, 0.99, 0.94, and 1.00 respectively) (Table 1). It was greater than 0.90, thereby confirming the pre-specified English factor structure.

\subsection{Explanatory factor analysis (EFA)}

Analysis of the Hebrew MDS-UPDRS responses differed from the English ones in factor structure of isolated items and in the findings of several items with cross-loading on multiple factors (Table 3), as follows.

For Part I, in contrast to the English language version of the MDS-UPDRS, Sleep problems, Pain and other sensations loaded on Factor 2 instead of Factor 1, and Fatigue loaded on both Factors 1 and 2. Pain and other sensations fell on the same category of Anxious mood as well as the categories of Sleep problems and Fatigue.

For Part II, Eating tasks loaded on all three factors, and Doing hobbies and other activities fell on both Factors 1 and 3, while it fell only on Factor 3 in the English version.

In Part III, Speech and Facial expression loaded on Factor 7, and nine items loaded on more than one factor. Toe tapping, left foot fell on Leg agility of both right and left foot, while Toe tapping of the right foot fell on a different factor. On the other hand, Postural tremor of both hands loaded together with Resting tremor and Constancy of rest tremor. There were no differences for Factor 3 between the two versions. Factors 4 and 5 referred to the right hemibody and the left hemibody, respectively, however, the English version did not have the same body distribution as ours. Factor 6 also referred to tremor, and Factor 7 referred to Speech and Facial expression in our case, while it was specifically related to the legs in the English version.

Part IV was almost identical in both languages, with the main difference being that Time spent with dyskinesias did not load on any of the factors in the Hebrew version. Functional impact of fluctuations and Complexity of motor fluctuations also loaded on Factor 2 in the Hebrew Scale. 


\section{Discussion}

In order for the MDS-UPDRS to fully replace its predecessor, the UPDRS version 3, as the international gold standard tool for the assessment of PD patients, it is vital that properly tested translations are made available for use in non-English speaking countries. The global program currently includes 12 non-English validated editions, and other language teams are organized for Dutch, Portuguese and Thai versions. The Hebrew version of the MDSUPDRS was evaluated in the current study.

The overall factor structure of the Hebrew language version was consistent with that of the English language version, confirming its suitability for use. Few isolated item differences in factor loadings were identified in the EFA of the Hebrew version, some loading on more than one factor while others loaded on a different factor compared to English MDS-UPDRS. In Part I, for example, Sleep problems and Pain and other sensations loaded on Factor 2 instead of Factor 1, This could be explained by the likelihood that people who suffer from chronic pain have more difficulties in falling asleep and maintaining sleep throughout the night compared to people without pain. That being said, it is well recognized that daytime sleepiness is a common symptom in people with cognitive decline, so it could be expected that those two items had loaded together. The dissimilarities between the English and Hebrew tested patients might be explained by cultural differences, with English speakers referring to isolated Sleep problems and not those that are a result of pain, while the Hebrew speakers using a broader definition and context.

In Part II, Eating tasks loaded on all three factors, unlike the English version that loaded only on Factor 2. This can be explained by the probability that Saliva and drooling, Chewing and swallowing, Tremor and Freezing can negatively affect the patient's ability to eat without assistance. Doing hobbies and other activities fell on Factors 1 and 3 in the Hebrew version but only on Factor 3 in the English version. We believe that Walking and balance as well as Freezing might very well affect our patients' capability of doing and enjoying hobbies, so it would not be surprising that Doing hobbies and other activities fell under both factors. We believe that here too, the discrepancy is culturally based with English speakers relating to the emotional aspect of Doing hobbies and other activities, while He-brew speaking Israeli patients refer to the problem on a more practical manner.

In Part III, Toe tapping, left foot fell together with Leg agility of both right and left foot, unlike the English version in which Toe tapping, left foot fell together with right foot and Leg agility also of both foots. We did not perform a statistical analysis to see to what extent our patients had left or right PD predominance, but that finding might be explained by the majority of them having had disease onset on the left side. As for Postural tremor, we consider it reasonable that Postural tremor of both hands fell together with Resting tremor and Constancy of rest tremor as it did in the Hebrew language version, although not in the English language version.

Rating scales are used for patient assessment, follow-up and for making decisions in clinical practice as well as for research. Implementation of these scales in other languages requires validation in order to guarantee their usefulness [11]. Independent validation of health 
measures is necessary to confirm or reject the findings obtained by the developers of the instrument. Reliability, validity and responsiveness are key properties of a scale: they are indicative of its quality as a measurement instrument and they have to be carefully tested $[12,13]$. The present study was designed to develop a cross-cultural adaptation of the MDSUPDRS in order to achieve the official Hebrew version of this scale. The main contributions of the study were: (1) comparison with the original study; (2) expansion of knowledge on clinimetric properties of the scale never before explored; and (3) availability of the MDSUPDRS for use in Hebrew-speaking settings.

This study has a number of limitations related to potential sample selection bias, since the data come from a single-center specialized clinical unit. In addition, the distribution of patients showed a predominance of patients in early/intermediate Hoehn and Yahr stages, with very few patients in stage 5 (a recognized characteristic of clinical samples).

Furthermore, it should be acknowledged that the cultural differences may affect the patients attitude toward motor and non motor symptoms.

\section{Conclusions}

The present study succeeded to develop a cross-cultural adaptation of the MDS-UPDRS to a Hebrew version of this scale. The overall factor structure of the Hebrew language version was consistent with that of the English language version, confirming its suitability for use. Further validation of the Hebrew MDS-UPDRS should be performed in the future to improve reliability.

The MDS-UPDRS Hebrew version reached the criteria to be designated as the Official Hebrew Version of the MDS-UPDRS.

\section{Acknowledgments}

\section{Grants/research}

NIH grants (R01NS091307, 5U01NS043127), grants from CHDI Foundation, Parkinson Disease Foundation and International Parkinson and Movement Disorder Society.

\section{References}

1. Chaudhuri KR, Healy D, Schapira AH. National Institute for Clinical Excellence. The non-motor symptoms of Parkinson's disease. Diagnosis and management. Lancet Neurol. 2006; 5:235-245. [PubMed: 16488379]

2. Ramaker C, Marinus J, Stiggelbout AM, van Hilten BJ. Systematic evaluation of rating scales for impairment and disability in Parkinson's disease. Mov Disord. 2002; 17:867-876. [PubMed: 12360535]

3. Fahn S, Elton RL. Unified Parkinson's disease rating scale. Recent Developments in Parkinson's Disease: MacMillan Healthcare Information. 1987:153-164.

4. Movement disorder society task force on rating scales for Parkinson's disease, The unified Parkinson's disease rating scale (UPDRS): status and recommendations. Mov Disord. 2003; 18:738750. [PubMed: 12815652]

5. Goetz CG, Tilley BC, Shaftman SR, et al. Movement Disorders Society sponsored revision of the Unified Parkinson's Disease Rating Scale (MDS-UPDRS): scale presentation and clinimetric testing results. Mov Disord. 2008; 23:2129-2170. [PubMed: 19025984] 
6. Goetz CG, Fahn S, Martinez-Martin P, et al. Movement disorder society-sponsored revision of the unified Parkinson's disease rating scale (MDS-UPDRS): process, format, and clinimetric testing plan. Mov Disord. 2006; 22:41-47.

7. Hoehn MM, Yahr MD. Parkinsonism: onset, progression, and mortality. Neurology. 1967; 17:427442. [PubMed: 6067254]

8. Marin PM, Blazquez CR, Sanchez MA, et al. Expanded and independentvalidation of the movement disorder society- Unified Parkinson's disease rating scale (MDS-UPDRS). J Neurol. 2013; 260:228236. [PubMed: 22865238]

9. Goetz CG, Stebbins GT, Wang L, Lapelle NR, Luo S, Tilley BC. IP-MDS sponsored scale translation program:Process, format, and clinimetric testing plan for the MDS-UPDRS and UDysRS. Mov Disord Clin Pract. 2014; 1:97-101. [PubMed: 27747259]

10. Gorsuch, R. Factor Analysis. 2. Lawrence Erlbaum Associations; Hillsdale: 1983.

11. Grove, A., Amante, PG., Quarterman, P., Wild, D. The translation and cross-cultural adaptation of quality-of-life measures. In: Jenkinson, C.Fitzpatrick, R., Jenkinson, D., editors. Health Status Measurement in Neurological Disorders. Radcliffe Medical Press; Oxon: 2000. p. 161-190.

12. Masur, H. Scales and Scores in Neurology. Thieme; Stuttgart: 2004. p. 4-51.

13. McDowell, I. A Guide to Rating Scales and Questionnaires. 3. Oxford University Press; Oxford: 2006. Measuring Health; p. 3-54. 
Table 1

Acceptability and reliability of the Hebrew version of the MDS-UPDRS.

\begin{tabular}{|c|c|c|c|c|}
\hline & Part I & Part II & Part III & Part IV \\
\hline Data quality (\%) & 97.68 & 98.71 & 94.34 & 98.23 \\
\hline Floor effect $(\%)$ & 0.80 & 0.5 & 0 & 46.10 \\
\hline Ceiling effect (\%) & 0 & 0 & 0 & 0 \\
\hline Skewness & 0.66 & 0.51 & 0.94 & 0.95 \\
\hline Cronbach's alpha & 0.79 & 0.90 & 0.93 & 0.80 \\
\hline Item total correlation & $0.18-0.60$ & $0.18-0.79$ & $0.25-0.71$ & $0.35-0.78$ \\
\hline Interclass correlation coefficient & 0.96 & 0.98 & 0.94 & 0.99 \\
\hline
\end{tabular}

MDS-UPDRS Movement Disorder Society-Unified Parkinson's Disease Rating Scale. 


\section{Table 2}

Characteristics of the study population.

\begin{tabular}{lcccl}
\hline & Number & Mean & SD & Range \\
\hline A. Demographics & & & & \\
Age, years & 389 & 69.09 & 9.37 & $40.00-93.00$ \\
Age at PD onset, years & 389 & 60.71 & 11.21 & $29.00-87.00$ \\
Duration of PD, years & 389 & 8.37 & 6.16 & $0.50-30.00$ \\
H\&Y stage & 388 & 2.45 & 1.01 & $1.00-5.00$ \\
Years of education & 383 & 14.34 & 3.81 & $0.00-30.00$ \\
B. MDS-UPDRS Results & 380 & 11.94 & 7.10 & $0.00-36.00$ \\
Part I & & & & \\
Non-motor aspects of EDL-total score & & & \\
Part II & 384 & 17.38 & 10.95 & $0.00-48.00$ \\
Motor aspects of EDL & & & & \\
Part III & 367 & 32.75 & 18.73 & $2.00-97.00$ \\
Motor examination & & & & \\
Part IV & 382 & 3.72 & 4.56 & $0.00-19.00$ \\
Motor complications & & & & \\
\hline
\end{tabular}

$E D L$ Experiences of daily living; $M D S$-UPDRS Movement Disorder Society-Unified Parkinson's Disease Rating Scale; $P D$ Parkinson's disease; $S D$ Standard deviation. 
Table 3

Comparison of English language and Hebrew exploratory factor structures for Parts I - IV of the MDSUPDRS.

\begin{tabular}{|c|c|c|c|}
\hline Factor & Item & \multicolumn{2}{|c|}{ Item factor loading } \\
\hline \multicolumn{4}{|c|}{ Part I: Non-Motor Aspects of Experiences of Daily Living } \\
\hline \multicolumn{4}{|c|}{$($ Section $\mathrm{CFI}=0.96, \mathrm{RSMEA}=0.06)$} \\
\hline & & English ${ }^{*}$ & Hebrew \\
\hline \multirow[t]{10}{*}{ Factor 1} & Percent variance & 34.0 & 34.2 \\
\hline & Daytime sleepiness & 0.53 & 0.44 \\
\hline & Sleep problems & 0.35 & $\operatorname{xxxx}$ \\
\hline & Cognitive impairment & 0.55 & 0.42 \\
\hline & Pain and other sensations & 0.43 & $\operatorname{xxxx}$ \\
\hline & Hallucinations and psychosis & 0.56 & 0.68 \\
\hline & Urinary problems & 0.61 & 0.48 \\
\hline & Constipation problems & 0.46 & 0.47 \\
\hline & Light headedness on standing & 0.46 & 0.43 \\
\hline & Fatigue & 0.47 & $0.41^{* * *}$ \\
\hline \multirow[t]{7}{*}{ Factor 2} & Percent variance & 9.5 & 10.1 \\
\hline & Depressed mood & 0.81 & 0.85 \\
\hline & Anxious mood & 0.68 & 0.69 \\
\hline & Apathy & 0.55 & 0.61 \\
\hline & Sleep problems & $\operatorname{xxxx}$ & 0.42 \\
\hline & Pain and other sensations & $\operatorname{xxxx}$ & 0.41 \\
\hline & Fatigue & $\operatorname{xxxx}$ & $0.57^{* * *}$ \\
\hline
\end{tabular}

Part II: Motor Aspects of Experiences of Daily Living

$($ Section CFI $=0.99$, RSMEA $=0.07)$

\begin{tabular}{llll}
\hline & & & \\
& & English $^{*}$ & Hebrew \\
\hline \multirow{2}{*}{ Factor 1} & Percent variance & 53.1 & 57.2 \\
& Speech & 0.79 & 0.89 \\
& Saliva and drooling & 0.45 & 0.48 \\
& Chewing and swallowing & 0.6 & 0.49 \\
& Handwriting & 0.46 & 0.52 \\
& Doing hobbies and other activities & 0.46 & $0.43^{* * * *}$ \\
& Eating tasks & & $0.35^{* * * *}$ \\
Factor 2 & Percent variance & xxx & 9.8 \\
& Eating tasks & 8.7 & $0.19^{* * * *}$
\end{tabular}




\begin{tabular}{lllc}
\hline Factor & Item & \multicolumn{2}{c}{ Item factor loading } \\
\hline Factor 3 & Percent variance & 7.7 & 7.7 \\
& Dressing & 0.64 & 0.88 \\
& Hygiene & 0.65 & 0.86 \\
& Turning in bed & 0.65 & 0.75 \\
& Getting out of bed & 0.73 & 0.80 \\
& Walking and balance & 0.82 & 0.76 \\
& Freezing & 0.76 & 0.61 \\
& Doing hobbies and other activities & $\mathrm{xxxx}$ & $0.60^{* * * *}$ \\
& Eating tasks & $\mathrm{xxxx}$ & $0.86^{* * * *}$ \\
\hline
\end{tabular}

Part III: Motor Examination

$($ Section CFI $=0.94$, RSMEA $=0.07)$

\begin{tabular}{|c|c|c|c|}
\hline & & English $^{*}$ & Hebrew \\
\hline \multirow[t]{12}{*}{ Factor 1} & Percent variance & 36.7 & 37.4 \\
\hline & Speech & 0.6 & $0.47^{* * *}$ \\
\hline & Facial expression & 0.54 & $0.40^{* * *}$ \\
\hline & Arising from chair & 0.8 & 0.79 \\
\hline & Gait & 0.87 & 0.85 \\
\hline & Freezing of gait & 0.83 & 0.72 \\
\hline & Postural stability & 0.8 & 0.80 \\
\hline & Posture & 0.7 & 0.81 \\
\hline & Global spontaneity of movement & 0.67 & 0.59 \\
\hline & Toe tapping, left foot & $\operatorname{xxxx}$ & $0.53^{* * *}$ \\
\hline & Leg agility, right leg & $\mathrm{xxxx}$ & $0.46^{* * *}$ \\
\hline & Leg agility, left leg & $\mathrm{xxxx}$ & $0.57^{* * *}$ \\
\hline \multirow[t]{9}{*}{ Factor 2} & Percent variance & 15.3 & 15.0 \\
\hline & Rest tremor amplitude, RUE & 0.73 & 0.75 \\
\hline & Rest tremor amplitude, LUE & 0.71 & 0.71 \\
\hline & Rest tremor amplitude, RLE & 0.74 & 0.80 \\
\hline & Rest tremor amplitude, LLE & 0.7 & 0.72 \\
\hline & Rest tremor amplitude, lip/jaw & 0.6 & 0.65 \\
\hline & Constancy of rest tremor & 0.88 & 0.83 \\
\hline & Postural tremor, right hand & $\operatorname{xxxx}$ & $0.65^{* * *}$ \\
\hline & Postural tremor, left hand & $\operatorname{xxxx}$ & $0.63^{* * *}$ \\
\hline \multirow[t]{5}{*}{ Factor 3} & Percent variance & 6.6 & 7.5 \\
\hline & Rigidity, neck & 0.68 & 0.66 \\
\hline & Rigidity, RUE & 0.73 & $0.61^{* * *}$ \\
\hline & Rigidity, LUE & 0.74 & 0.63 \\
\hline & Rigidity, RLE & 0.8 & $0.65^{* * * *}$ \\
\hline
\end{tabular}




\begin{tabular}{|c|c|c|c|}
\hline \multirow[t]{2}{*}{ Factor } & \multirow{2}{*}{$\frac{\text { Item }}{\text { Rigidity, LLE }}$} & \multicolumn{2}{|c|}{ Item factor loading } \\
\hline & & 0.82 & 0.68 \\
\hline \multirow[t]{9}{*}{ Factor 4} & Percent variance & 6.2 & 5.6 \\
\hline & Finger tapping, right hand & 0.67 & 0.73 \\
\hline & Hand movements, right hand & 0.67 & 0.81 \\
\hline & Pronation/supination, right hand & 0.7 & 0.73 \\
\hline & Rigidity, RUE & $\operatorname{xxxx}$ & $0.46^{* * *}$ \\
\hline & Rigidity, RLE & $\operatorname{xxxx}$ & $0.41^{* * * *}$ \\
\hline & Toe tapping, right foot & $\mathrm{xxxx}$ & 0.68 \\
\hline & Leg agility, right leg & $\operatorname{xxxx}$ & $0.72^{* * *}$ \\
\hline & Rest tremor amplitude, RUE & $\operatorname{xxxx}$ & 0.43 \\
\hline \multirow[t]{7}{*}{ Factor 5} & Percent variance & 4.9 & 4.7 \\
\hline & Finger tapping, left hand & 0.67 & 0.59 \\
\hline & Hand movements, left hand & 0.7 & 0.69 \\
\hline & Pronation/supination movements, left hand & 0.65 & 0.68 \\
\hline & Rigidity, LUE & $\operatorname{xxxx}$ & 0.41 \\
\hline & Toe tapping, left foot & $\operatorname{xxxx}$ & $0.57^{* * *}$ \\
\hline & Leg agility, left leg & $\operatorname{xxxx}$ & $0.52^{* * *}$ \\
\hline \multirow[t]{5}{*}{ Factor 6} & Percent variance & 4.5 & 4.5 \\
\hline & Postural tremor, right hand & 0.66 & $0.45^{* * *}$ \\
\hline & Postural tremor, left hand & 0.71 & $0.42^{* * * *}$ \\
\hline & Kinetic tremor, right hand & 0.81 & 0.66 \\
\hline & Kinetic tremor, left hand & 0.81 & 0.56 \\
\hline \multirow[t]{7}{*}{ Factor 7} & Percent variance & 3.3 & 3.4 \\
\hline & Toe tapping, right foot & 0.65 & $\operatorname{xxxx}$ \\
\hline & Toe tapping, left foot & 0.62 & $\operatorname{xxxx}$ \\
\hline & Leg agility, right leg & 0.62 & $\operatorname{xxxx}$ \\
\hline & Leg agility, left leg & 0.6 & $\operatorname{xxxx}$ \\
\hline & Speech & $\mathrm{xxxx}$ & $0.62^{* * *}$ \\
\hline & Facial expression & $\operatorname{xxxx}$ & $0.63^{* * *}$ \\
\hline
\end{tabular}

Part IV: Motor Complications

$($ Section CFI $=1.00$, RSMEA $=0.09)$

\begin{tabular}{llll}
\hline & & English ${ }^{*}$ & Hebrew \\
\hline Factor 1 & Percent variance & 63.9 & 71.5 \\
& Time spent in the OFF state & 0.87 & 0.89 \\
& Functional impact of fluctuations & 0.84 & $0.70^{* * *}$ \\
& Complexity of motor fluctuations & 0.82 & $0.65^{* * *}$ \\
& Painful OFF state dystonia & 0.5 & 0.57 \\
& Percent variance & 15.6 & 11.8
\end{tabular}

Parkinsonism Relat Disord. Author manuscript; available in PMC 2018 May 29. 


\begin{tabular}{|c|c|c|c|}
\hline \multirow{2}{*}{$\frac{\text { Factor }}{\text { Factor } 2}$} & \multirow{2}{*}{$\begin{array}{l}\text { Item } \\
\text { Time spent with dyskinesias }\end{array}$} & \multicolumn{2}{|c|}{ Item factor loading } \\
\hline & & 0.71 & 0.77 \\
\hline & Functional impact of dyskinesias & 0.95 & 0.94 \\
\hline & Functional impact of fluctuations & $\operatorname{xxxx}$ & $0.57^{* * *}$ \\
\hline & Complexity of motor fluctuations & $\operatorname{xxxx}$ & $0.49^{* * *}$ \\
\hline \multicolumn{4}{|c|}{ Because we are using a different version of M-Plus, the factor loadings may vary slightly from published version. } \\
\hline \multicolumn{4}{|c|}{ 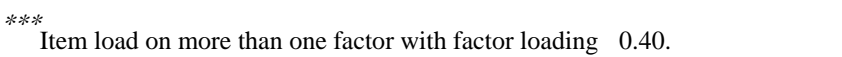 } \\
\hline
\end{tabular}

